\title{
ESTILO ATRIBUTIVO NEGATIVO, SUCESOS VITALES Y SINTOMATOLOGÍA DEPRESIVA
}

\author{
Pilar SanJuÁn y Alejandro Magallares \\ Universidad Nacional de Educación a Distancia, Madrid
}

\begin{abstract}
Resumen: El estilo atributivo negativo es una variable cognitiva de personalidad que refleja la tendencia relativamente estable a explicar los eventos negativos mediante causas internas, estables y globales. El modelo reformulado de indefensión aprendida y su desarrollo posterior, la tooría de la desesperanza, predicen que el estilo atributivo negativo interactúa con el estrés percibido en la predicción de sintomatología depresiva. El principal objetivo del presente estudio fue probar esta hipótesis con una muestra española de población adulta. Los resultados confirmaron la hipótesis. Se discute la conveniencia de estudiar algunos factores, como autoestima, percepción de controlabilidad sobre las situaciones estresantes o grado de importancia que se le da a las mismas, que pueden modular la relación entre este estilo explicativo y el desarrollo de sintomas depresivos, pero que han recibido muy poca atención hasta la fecha.
\end{abstract}

Palabras clave: Estilo atributivo negativo, sucesos vitales, sintomas depresivos.

\section{Negative attributional style, life events, and depressive symptomatology}

\begin{abstract}
Negative attributional style is a cognitive personality variable that reflects a stable tendency to explain negative events with causes that are internal to self, stable across time and global in effect. The reformulated learned helplessness model and its next development, the hopelessness theory, predict that negative attributional style interacts with perceived stress to predict depressive symptomatology. The first goal of the present study was to prove this hypothesis with an adult Spanish sample. The results confirmed the hypothesis. We discuss the convenience to study factors such as self-esteem, the perception of the controllability of stressful situations or the degree of their subjective importance. These factors may modulate the relationship between this explanatory style and the development of depressive symptoms, but have received very little attention up to now.
\end{abstract}

Keywords: Negative attributional style, life events, depressive symptoms.

\section{INTRODUCCIÓN}

Los informes sobre prevalencia ponen de relieve que la depresión es uno de los trastornos que está aumentando su incidencia en las sociedades occidentales. Aunque algunos estudios han mostrado que la sintomatologia depresiva se asocia con la ocurrencia de altos niveles de estres, éste soblo puede explicar entre un 5 y un $20 \%$ de la varianza de dicha sintomatologia. Por ello, la investigación se ha dirigido a identificar los posibles moderadores del efecto del estrés en el ini-

Recibido 20 mayo 2006; aceptado 30 junio 2006.

Correspondencia: Pilar Sanjuán, Departamento de Personalidad, Evaluación y Tratamiento Psicológicos, Facultad de Psicología de la UNED, c/ Juan del Rosal 10, 28040 Madrid (Bspafia): Correo-e: peanjuan@psi.uned.es cio y desarrollo de este tipo de problema. Toda esta investigación ha permitido descubrir la importancia de los factores cognitivos en el inicio, mantenimiento y tratamiento, tanto de la sintomatología depresiva, como de la depresión clfnica. En esta línea, una de las propuestas que más atención y apoyo está recibiendo en la actualidad es el modelo atribucional de vulnerabilidad cognitiva de la depresión.

La primera perspectiva que destacó la relación de los procesos atributivos con la sintomatología depresiva fue el modelo reformulado de indefensión aprendida (Abramson, Seligman y Teasdale, 1978) al considerar que la realización de una atribución es el paso previo a la formación de la expectativa de incontrolabilidad y el subsiguiente desarrollo de sintomas depresivos. Especificamente, el modelo propone que cuando las perso- 
nas son sometidas a experiencias de incontrolabilidad hacen una atribución respecto de cuál es la causa de esa falta de control. Esta atribución se hace en función de tres dimensiones: internalidadexternalidad (según que se atribuya el resultado a una causa debida al propio sujeto o externa a él), estabilidad-inestabilidad (en función de que se crea que la causa se mantendrá o no en el futuro) y globalidad-especificidad (de acuerdo con que se piense que la causa afectará a más áreas de la vida o sólo a la situación concreta).

Ya en este primer trabajo (Abramson et al., 1978) sugirieron que las personas difieren en la manera habitual en que explican las causas de las situaciones a las que se enfrentan. Las personas que tienen una tendencia relativamente estable a explicar los sucesos negativos mediante causas internas, estables y globales se dice que tienen un estilo explicativo negativo, mientras que las que tienden a explicarlos con causas externas, inestables y específicas se considera que emplean un estilo explicativo positivo. En este primer trabajo también apuntaron que las personas con un estilo explicativo negativo serían más vulnerables, y por ello, desarrollarian sintomatología depresiva con más facilidad cuando se enfrentaran con sucesos negativos en sus vidas.

La gran cantidad de estudios realizados desde el modelo reformulado de indefensión aprendida ha probado sobradamente que existe una relación entre el estilo atributivo negativo y la depresión, ya sea clínica o subclínica, o el estado de ánimo negativo (véase Sanjuán, 1999; Sweeney, Anderson y Bailey, 1986; para una revisión). Sin embargo, aunque lo que se defendía es una interacción, más que un efecto principal del estilo explicativo negativo, de tal manera que en ausencia de estrés, no se espera que las personas con este estilo atributivo incrementen su riesgo de desarrollar sintomas depresivos, desde esta perspectiva no se han realizado estudios que permitan probarla.

La investigación que intenta probar la hipótesis sobre el papel que juega la interacción entre este estilo atributivo y la ocurrencia de situaciones estresantes en la aparición de sintomatología depresiva se ha abordado desde un desarrollo del modelo anterior que se conoce como Teoría de la Desesperanza (Abramson, Metalsky y Alloy, 1989; Abramson, Alloy y Metalsky, 1995; Abramson, Alloy, Metalsky, Joiner y Sandín, 1997). Desde esta teoría cognitiva sí se han realizado muchos trabajos para probar la hipótesis diátesis-estrés, que es como se conoce la interacción postulada entre el estilo atributivo negativo (diatesis o predisposición) y la ocurrencia de situaciones negativas (estrés) que predeciría el inicio y desarrollo de la sintomatología depresiva.

Desde este marco teórico, los estudios que se han llevado a cabo han utilizado fundamentalmente dos estrategias para valorar el estrés, o emplear un fracaso académico real o medir la ocurrencia de diversos sucesos vitales negativos a través de cuestionarios. En general, la investigación al respecto ha confirmado la hipótesis planteada, encontrándose que las personas con un estilo explicativo negativo, con respecto a las que tienen un estilo explicativo positivo, presentan una mayor sintomatología depresiva cuando se enfrentan con situaciones estresantes, obteniéndose resultados similares tanto si se trata de un fracaso académico real (Hilsman y Garber, 1995; Metalsky, Halberstadt y Abramson, 1987; Stiensmeier-Pelster, 1989; Vázquez, Jiménez, Saura y Avia, 2001), como si se valora la ocurrencia de sucesos vitales negativos (Abela, 2001; Alloy et al., 1999; Alloy y Clements, 1998; Alloy, Just y Panzarella, 1997; Dixon y Ahrens, 1992; Hankin y Abramson, 2002; Hankin, Abramson y Siler, 2001; Kneebone y Dunmore, 2004; Know y Laurenceau, 2002; Metalsky y Joiner, 1992, 1997; Stiensmeier-Pelster, 1989).

Otros estudios realizados desde esta perspectiva, aunque muy pocos en número, en comparación con los anteriores, no han obtenido los resultados esperables de acuerdo con la teoría. Así, el estudio de Follette y Jacobson (1987) encontró que el estilo explicativo negativo no era el que predecía el incremento de síntomas depresivos después de un fracaso académico, sino las atribuciones concretas que se realizaban ante ese fracaso en particular. Los resultados del trabajo de Herrera y Maldonado (2002) mostraron que lo que predice el nivel de depresión después de un fracaso en un examen es el nivel previo de la misma y no el estilo atributivo negativo. En la investigación de Ralph y Mineka (1998) el estilo atributivo negativo predecía el incremento de ansiedad, pero no de síntomas depresivos después de un fracaso académico. De la misma manera, los resultados del estudio de Lewinsohn, Joiner y Rohde (2001) sugieren que existe un umbral de vulnerabilidad a la depresión para aquellos que han experi- 
mentado sucesos vitales negativos, en el sentido de que el inicio de los episodios de depresión mayor se relaciona con actitudes disfuncionales previas sólo cuando éstas exceden un determinado nivel, apuntando, por tanto, la necesidad de tener en cuenta otros factores además del estilo atributivo negativo.

El objetivo fundamental del presente estudio fue probar la hipótesis diátesis-estrés utilizando una población española adulta y evaluando la ocurrencia de sucesos vitales negativos. Los estudios realizados con población española son muy escasos y, además, se han limitado a emplear fracasos académicos (Herrera y Maldonado, 2002; Vázquez et al., 2001), pero ninguno ha evaluado la incidencia de sucesos vitales estresantes o negativos.

\section{MÉTODO}

\section{Participantes}

Se empleó una muestra formada por 188 estudiantes universitarios, 42 varones y 146 mujeres, con un rango de edad entre 19 y 61 años y con una media de 35,7 años (desviación típica $=10,7$ ).

\section{Medidas y procedimiento}

Los participantes contestaron en una única sesión a las siguientes medidas:

Estilo Atributivo. Se evaluó con la adaptación española (Palomares y Sanjuán, 1995; Sanjuán y Magallares, 2005) del Cuestionario de Estilo Atribucional (Attributional Style Questionnaire: ASQ, Peterson et al., 1982). Este cuestionario consta de 12 situaciones hipotéticas, 6 con un resultado positivo y 6 con resultado negativo, la mitad de cada una de ellas, a su vez, se refieren a situaciones de logro, mientras que la otra mitad hace alusión a situaciones interpersonales o afiliativas. Ante cada una de estas situaciones, el sujeto contesta mediante escalas tipo Likert de 7 puntos a 4 preguntas: el grado en que cree que la situación es debida a factores internos, estables y globales, así como el nivel de importancia de la situación, indicando el «l» que considera que la situación es totalmente debida a factores externos, inestables y específicos, y nada importante, y el «7» que piensa que la situación es producida por factores totalmente internos, estables y globales, y que es muy importante para la persona. Con esta escala se puede obtener una puntuación por cada una de las dimensiones atributivas (internalidad, estabilidad y globalidad) tanto para los sucesos negativos como para los positivos separadamente, así como dos puntuaciones totales, denominadas estilos compuestos (negativo y positivo). Para los propósitos de la investigación sólo se utilizó el estilo compuesto negativo ( $E C N$ ), que seria un indice del estilo atribucional presentado ante los sucesos negativos. Las puntuaciones más altas en ECN señalarian la tendencia a emplear causas internas, estables y globales para explicar las situaciones negativas (lo que hemos denominado estilo atributivo negativo) y las más bajas la predisposición a explicarlas mediante causas externas, inestables y especificas (al que se llama estilo atributivo positivo). En el presente estudio el coeficiente de consistencia interna del ECN fue de 0,79.

Sucesos vitales. Se midieron con el Cuestionario de Sucesos Vitales de Sandín (1999), que consta de 66 items en los que se recogen eventos de distintas áreas (trabajo, salud, amor, pareja, familia, hijos, social, legal, finanzas, residencia y académica). El cuestionario permite conocer el número de sucesos vitales ocurridos, así como el grado de estrés percibido o intensidad del estrés de cada uno de estos sucesos, mediante escalas tipo Likert de 4 puntos, en las que el «l» indica nada de intensidad y el «4» muchisima. También es posible obtener puntuaciones diferenciadas para los eventos considerados positivos y negativos. En este estudio los participantes informaron de los sucesos a los que se habían enfrentado en el último mes, y sólo se analizaron los que consideraron negativos. Los datos del presente trabajo arrojaron un coeficiente de consistencia interna de 0,59 , que es similar a los encontrados en los estudios de baremación de la escala (Sandín, 1999).

Sintomatología depresiva. Se evaluó con la adaptación española (Sanz y Vázquez, 1998; Vázquez y Sanz, 1997) del Inventario de Depresión de Beck (Beck Depression Inventory: BDI; Beck, Rush, Shaw y Emery, 1979). Este cuestionario consta de 21 items, que reco- 
gen los síntomas cognitivos, afectivos, motivacionales y fisiológicos de la depresión. Cada uno de los items puede tener $4,5,6$ u 8 alternativas, que se puntúan entre 0 y 3 dependiendo de la severidad de los síntomas a los que hace referencia. Es uno de los instrumentos más empleados para la medida de la depresión, por lo que se ha corroborado en reiteradas ocasiones su fiabilidad y validez. Con los datos de nuestro estudio se obtuvo un coeficiente de consistencia interna de $\mathbf{0 , 8 1}$.

\section{RESULTADOS}

Antes de comenzar con los análisis estadísticos encaminados a descubrir las posibles relaciones establecidas entre las distintas variables, realizamos pruebas de $t$ para comprobar si existían diferencias entre varones y mujeres en cada una de las variables de interés empleadas en el estudio (estilo atributivo, sucesos vitales o nivel de estrés y estado de ánimo depresivo). Ninguna de estas pruebas fue significativa (todas las $p>$ 0,6 ), debido a lo cual, en los análisis posteriores se incluyó a todos los sujetos de la muestra independientemente de su sexo.

En la Tabla 1 aparecen los estadísticos descriptivos y correlaciones de las distintas variables que se han empleado en el estudio, después de calcular

Tabla 1. Estadísticos descriptivos y correlaciones de las variables del estudio

\begin{tabular}{lccc}
\hline & ECN & Estrés & BDI \\
\hline ECN & - & & \\
Estrés & 0,09 & - & \\
BDI & $0,27 *$ & $0,21^{*}$ & - \\
Media & 3,93 & 2,68 & 4,76 \\
DT & 0,63 & 0,75 & 5,31 \\
\hline
\end{tabular}

Nota: ECN: Estilo Compuesto Negativo; BDI: Inventario de Depresión de Beck. ${ }^{*} p<0,001$. la variable a la que denominamos nivel de estrés que es el resultado de dividir la intensidad del estrés percibido en los sucesos vitales negativos entre la frecuencia de sucesos vitales ocurridos.

Para probar la hipótesis de que el estilo atributivo para los sucesos negativos interactúa con la ocurrencia de sucesos vitales estresantes para producir sintomatología depresiva, empleamos el procedimiento estadístico que utilizan la gran mayoría de los estudios llevados a cabo dentro de este marco teórico (Abela, 2001; Alloy y Clements, 1998; Hankin et al., 2001; Hilsman y Garber, 1995; Metalsky et al., 1987; Robinson, Garber y Hilsman, 1995; Southall y Roberts, 2002; Stiensmeier-Pelster, 1989) consistente en realizar un análisis de regresión múltiple jerárquico (Cohen y Cohen, 1983). La variable criterio fue la sintomatología depresiva (puntuación en el BDI) y las variables predictoras, el estilo atributivo (estilo compuesto negativo: ECN) y el nivel de estrés.

Como puede observarse en la Tabla 2, ambas variables son predictores significativos de la sintomatología depresiva, explicando mayor porcentaje de varianza la interacción de ambos, que la de cada uno por separado (beta-estrés $=0,39 \mathrm{y}$ beta$\mathrm{ECN}=0,26$; ambas significativas con $p<0,000$ ).

Siguiendo el procedimiento recomendado por los investigadores a los que se ha hecho referencia anteriormente, se realizó un análisis de varianza de dos factores (ECN y estrés), manipulados entresujetos, utilizando la sintomatología depresiva como variable dependiente. Para lo cual se formaron previamente grupos extremos en las dos variables predictoras (ECN y estrés), siguiendo el criterio de seleccionar a las personas que se encontraban por encima del tercer cuartil (altos en ECN o personas con estilo explicativo negativo y altos en estrés, respectivamente) o por debajo del primero (bajos en ECN o personas con estilo explicativo positivo y bajos en estrés, respectivamente).

$E l$ análisis de los resultados, que pueden verse en la Tabla 3, arrojó efectos principales signi-

Tabla 2. Resuitados del análisis de regresión múltiple jerárquico utilizando como criterio la puntuación en el BDI

\begin{tabular}{ccccccc}
\hline Pasos & Predictor añadido & $R$ & $R^{2}$ & $\Delta R^{2}$ & $F$ & g.1. \\
\hline 1 & Estrés & 0,4 & 0,16 & 0,16 & $35,67^{*}$ & 1,187 \\
2 & ECN & 0,48 & 0,23 & 0,07 & $27,46^{*}$ & 2,186 \\
\hline
\end{tabular}

$p<0,000$ 
Tabla 3. Medias (y desviaciones típicas) en el BDI en función del estilo atributivo y el nivel de estrís

\begin{tabular}{lccc}
\hline & Estilo Atributivo Positivo & Estilo Atributivo Negativo & Total \\
\hline Bajo estrés & $3,6(3,37)$ & $5,36(4,54)$ & $4,52(4,3)$ \\
Alto estrés & $2,83(3,74)$ & $12,25(8,92)$ & $6,6(7,73)$ \\
\hline Total & $3,18(3,51)$ & $8,26(7,39)$ & \\
\hline
\end{tabular}

ficativos del estilo atributivo $[F(1,41)=11,36$, $p<0,002]$ y del estrés $[F(1,41)=3,4, p<$ $0,007]$, lo que indica, por un lado, que las personas con un estilo explicativo negativo presentan una mayor sintomatología depresiva que los que tienen un estilo explicativo positivo y, por otro, que los participantes que sufrieron un alto grado de estrés también puntuaron más en estado de ánimo negativo que los que pasaron por niveles bajos de estrés. A la vista de los resultados mostrados en la tabla parece evidente que estos efectos se deben a la interacción significativa existente entre los dos factores $[F(1,41)=5,32, p<$ $0,02]$, que a su vez se explica porque en la condición de alto estrés, las personas con un estilo explicativo negativo informaron de más sintomas depresivos que las que tienen un estilo explicativo positivo $\left(t_{18}=-3,28, p<0,004\right)$. Además, los participantes con un estilo atributivo negativo presentaron mayor sintomatología depresiva cuando habían estado sometidos a altos niveles de estrés que cuando el estrés había sido muy bajo $\left(t_{17}=-2,21, p<0,04\right)$.

\section{DISCUSIÓN}

Los resultados obtenidos ponen de manifiesto que en el grupo de personas con un estilo explicativo negativo y que además informan haber estado sometidos a un alto grado de estrés, es en el que se presenta una mayor sintomatología depresiva. Esta clara interacción entre el estilo atributivo negativo y el estrés, corrobora la hipótesis diátesis-estrés apuntada desde el modelo reformulada de indefensión aprendida (Abramson et al., 1978) y puesta a prueba en numerosos estudios realizados desde la Teoría de la Desesperanza (Abela, 2001; Alloy et al., 1999; Alloy y Clements, 1998; Alloy et al., 1997; Dixon y Ahrens, 1992; Hankin y Abramson, 2002; Hankin et al., 2001; Kneebone y Dunmore, 2004; Know y Laurenceau, 2002; Metalsky y Joiner, 1992, 1997; Stiensmeier-Pelster, 1989). Los resultados ponen de relieve cómo las personas con un estilo explicativo negativo sólo alcanzan niveles elevados de sintomas depresivos cuando han estado sometidos a altos grados de estrés, pero no cuando el estrés ha sido muy bajo. De esta manera parece confirmarse que este estilo explicativo es un factor de vulnerabilidad, que sólo actuaria cuando la persona se encuentra sometida a situaciones estresantes, permaneciendo latente en situaciones neutras. Por otro lado, si tomamos en consideración los resultados relativos a las personas con un estilo explicativo positivo, parecen sugerir un cierto rol protector, puesto que en situaciones, tanto de alto como de bajo estrés, informan los menores niveles de sintomas depresivos.

Los resultados obtenidos son muy claros, sin embargo, es necesario seguir realizando trabajos en esta línea de investigación que puedan superar la principal limitación de este estudio, que se deriva de su naturaleza transversal. Los estudios longitudinales son los únicos capaces de probar que el estilo atributivo negativo es realmente un factor de vulnerabilidad para desarrollar sintomatologia depresiva cuando las personas se enfrentan con situaciones estresantes, pues de otra manera es imposible llegar a dilucidar si el estilo atributivo es una causa o una consecuencia del estado de ánimo negativo.

De cara a futuros trabajos, también sería interesante incluir medidas de carácter más objetivo que los meros autoinformes, que están sujetos a ciertos sesgos (Garcia y Belloch, 2004; Rueda y Pérez-García, 2004).

De la misma manera, consideramos que en estudios posteriores sería conveniente incluir ciertas variables relevantes, que podrían ayudar a explicar los resultados discrepantes obtenidos en algunos estudios de otros autores (Cole y Turner, 1993; Follette y Jacobson, 1987; Herrera y Maldonado, 2002; Lewinsohn et al., 2001; Ralph y Mineka, 1998), pero que, sin embargo, han recibido muy poca atención hasta la fecha por parte de los investigadores. Despues de revisar los estudios empiricos sobre esta problemática, se podría apuntar que 
las variables cuyo estudio ayudarían a dilucidar la cuestión serían, por un lado, la autoestima, el grado de importancia que se da a los sucesos negativos y el nivel de controlabilidad de los mismos. Por otra parte, el estudio de la ansiedad y la afectividad negativa como variables criterio, además de la depresión, también podría ser esclarecedor.

En cuanto a la necesidad de analizar la autoestima, algunos trabajos han puesto de relieve que el estilo atributivo negativo predice el incremento de estado de ánimo negativo después de un fracaso (Metalsky, Joiner, Hardin y Abramson, 1993) o de la ocurrencia de sucesos vitales estresantes (Robinson et al., 1995; Southall y Roberts, 2002), pero sólo entre las personas que tienen autoestima baja.

Por lo que respecta al grado de importancia que se da a las situaciones negativas, hay que señalar que la teoria propone que sólo los sucesos valorados como importantes por los individuos serían relevantes a la hora de predecir el desarrollo de los síntomas, sin embargo, la realidad es que es una variable que sistemáticamente se omite de los análisis. El estudio de Vázquez et al. (2001) ha puesto de relieve que es una variable que se tiene que considerar puesto que encontró que las mayores puntuaciones en depresión se producian entre los individuos que tenían un estilo atributivo negativo para situaciones relacionadas con el logro, valoraban esos sucesos como importantes, fracasaban en un examen y juzgaban ese fracaso como muy importante.

La expectativa de incontrolabilidad, que formalmente ocupaba un rol central en la teoría, gradualmente se ha ido reemplazando por el de situaciones negativas o estresantes, utilizándose en la literatura como sinónimos. Sin embargo, los sucesos negativos pueden ser controlables o incontrolables y algunos autores han sugerido que sólo los sucesos que se explican mediante causas incontrolables se asocian con el desarrollo de síntomas depresivos o depresión (Janoff-Bulman, 1979).

Para terminar con nuestra línea de argumentación a favor de la inclusión de determinadas variables, habria que decir que, aunque el estilo atributivo negativo comenzó estudiándose en relación con la depresión o el estado de ánimo negativo, algunos estudios le han asociado con ansiedad, en sus múltiples facetas de rasgo, estado o trastorno clínico (Helton, Dember, Warm y Matthews, 2000; Jackson, Sellers y Peterson, 2002; Kopocky, Sawyer y Behnke, 2004; MartinKrumm, Sarrazin, Peterson y Famose, 2003;
Mineka, Pury y Luten, 1995; Ralph y Mineka, 1998; Wang y Zhang, 2005). Debido a estos resultados, algunos autores han sugerido que el estilo atributivo no se asociaría específicamente con depresión, sino con una alta emocionalidad negativa, que sería una característica que compartirían tanto la depresión como la ansiedad (Ahrens y Haaga, 1993; Mineka et al., 1995).

\section{REFERENCIAS}

Abela, J. (2001). The hopelessness theory of depression: A test of the diathesis-stress and causal mediation components in third and seventh grade children. Journal of Abnormal Child Psychology, 29, 241-254.

Abramson, L.Y., Alloy, L.B., y Metalsky, G.I. (1995). Hopelessness depression. En G.M. Buchanan y M.E.P. Seligman (Eds.), Explanatory Style (pp. 113-134) Hillsdale, N.J.: Erlbaum.

Abramson, L.Y., Alloy, L.B., Metalsky, G.I., Joiner, T.E., y Sandín, B. (1997). Teoría de la depresión por desesperanza: Aportaciones recientes. Revista de Psicopatologia y Psicologia Clínica, 2, 211-222.

Abramson, L.Y., Metalsky, G.I., y Alloy, L.B. (1989) Hopelessness depression: A theory-based subtype of depresión. Psychological Review, 96, 358-372

Abramson, L.Y., Seligman, M.E.P., y Teasdale, D.C. (1978). Learned helplessness in humans: Critique and reformulation. Journal of Abnormal Psychology, 87, 49-74.

Ahrens, A. H., y Haaga, D.A.F. (1993). The specificity of attributional style and expectations to positive and negative affectivity, depression and anxiety. Cognitive Therapy and Research, 17, 83-98.

Alloy, L., Abramson, L.Y., Whitehouse, W., Hogan, M., Tashman, N.A., Steinberg, D., Rose, D., y Donovan, P. (1999). Depressogenic cognitive styles: Predictive validity, information processing and personality characteristics, and developmental origins. Behaviour Research and Therapy, 37, 503-531.

Alloy, L.B., y Clements, C.M. (1998). Hopelessness theory of depression: Tests of the symptom component. Cognitive Therapy and Research, 22, 303-335.

Alloy, L.B., Just, N., y Panzarella, C. (1997). Attributional style, daily life events, and hopelessness depression: Subtype validation by prospective variability and specificity of symptoms. Cognitive Therapy and Research, 21, 321-344.

Beck, A.T., Rush, A.J., Shaw, B.F, y Emery, G. (1979). Cognitive therapy of depression. Nueva York: Guilford Press.

Cohen, J., y Cohen, P. (1983). Applied multiple regression/correlation analysis for the behavional sciences. Hillsdale, N.J.: Erlbaum. 
Cole, D.A., y Turner, J.E. (1993). Models of cognitive mediation and moderation in child depression. Journal of Abnormal Psychology, 102, 271-281.

Dixon, J.F., y Ahrens, A.H. (1992). Stress and attributional style as predictor of self-reported depression in children. Cognitive Therapy and Research, 16, 623-634.

Follete, V., y Jacobson, N. (1987). Importance of attributions as a predictor of how people cope with failure. Journal of Personality and Social Psychology, 52, 1205-1211.

García, G., y Belloch, A. (2004). Influencia del estado de ánimo sobre la supresión de imágenes. Revista de Psicopatologia y Psicologia Clínica, 9, 183-203.

Hankin, B.L, y Abramson, L.Y. (2002). Measuring cognitive vulnerability in adolescence: Reliability, validity and gender differences. Journal of Clinical Child and Adolescent Psychology, 31, 491-504.

Hankin, B.L., Abramson, L.Y., y Siler, M. (2001). A prospective test of the hopelessness theory of depression in adolescence. Cognitive Therapy and Research, 25, 607632.

Helton, W.S., Dember, W.N., Warm, J.S., y Matthews, G. (2000). Optimism, pessimism, and false failure feedback effects on vigilance performance. Current Psychology, 18, 311-325.

Herrera, A., y Maldonado, A. (2002). Cognición y depresión ¿causa o efecto? Psicología Conductual, 10, 269. 285.

Hilsman, R., y Garber, J. (1995). A test the cognitive diathesis-stress model of depression in children: academic stressors, attributional style, perceived competence, and control. Journal of Personality and Social Psychology, 2, 370-380.

Jackson, B., Sellers, R.M., y Peterson, C. (2002). Pessimistic explanatory style moderates the effects of stress on physical illness. Personality and Individual Differences, $32,567-573$.

Janoff-Bulman, R. (1979). Characterological versus behavioral self-blame: Inquiries into depression and rape. Journal of Personality and Social Psychology, 37, 1798-1809.

Kneebone, 1.I., y Dunmore, E. (2004). Attributional style and symptoms of depression in persons with multiple sclerosis. International Journal of Behavional Medicine, 11, 110-115.

Kopecky, C., Sawyer, C., y Behnke, R. (2004). Sensitivity to punishment and explanatory style as predictors of public speaking state anxiety. Communication Education, 53, 281-285.

Kwon, P., y Laurenceau, J.P. (2002). A longitudinal study of the hopelessness theory of depression: Testing the diathesis-stress model within a differential reactivity exposure framework. Journal of Clinical Psychology, $58,1305-1321$.

Lewinsohn, P.M., Joiner, T.E., y Rohde, P. (2001). Evaluation of cognitive diathesis-stress models in predicting major depressive disorder in adolescents. Journal of Abnormal Psychology, 110, 203-215.

Martin-Krumm, C., Sarrazin, P., Peterson, C., y Famose, J. (2003). Explanatory style and resilience after sport failure. Personality and Individual Differences, 35 , 1685-1697.

Metalsky, G.I., Halberstadt, L.J., y Abramson, L.Y. (1987). Vulnerability to depressive mood reactions: Toward a more powerful test of the diathesis-stress and causal mediation components of the reformulated theory of depression. Journal of Personality and Social Psychology, 52, 386-393.

Metalsky, G.I., y Joiner, T.E. (1992). Vulnerability to depressive symptomatology: A prospective test of the diathesis-stress and causal mediation components of the hopelessness theory of depression. Journal of Personality and Social Psychology, 63, 667-675.

Metalsky, G.I., y Joiner, T.E. (1997). The Hopelessness Depression Symptom Questionnaire. Cognitive Therapy and Research, 21, 359-384.

Metalsky, G.I., Joiner, T.E., Hardin, T.S., y Abramson, L.Y. (1993). Depressive reactions to failure in a naturalistic setting: A test of the hopelessness and self-esteem theories of depression. Journal of Abnormal Psychology, 102, 101-109.

Mineka, S., Pury, C.L., y Luten, A.G. (1995). Explanatory style in anxiety and depression. En G.M. Buchanan y M.E.P. Seligman (Eds.), Explanatory style (pp. 135-157). Hillsdale, N.J.: Eribaum.

Palomares, A., y Sanjuán, P. (1995). A Spanish version of Attributional Style Questionnaire (ASQ). IV European Congress of Psychology. Atenas, 2-7 Julio. (Acta de Congreso: p. 442).

Peterson, C., Semmel, A., Baeyer, C., Abramson, L.Y., Metalsky, G.I., y Seligman, M.E.P. (1982). The Attributional Style Questionnaire. Cognitive Therapy and Research, 6, 287-300.

Ralph, J.A., y Mineka, S. (1998). Attributional style and self-esteem: The prediction of emotional distress following a midterm exam. Journal of Abnormal Psychology, 107, 203-215.

Robinson, N., Garber, J., y Hilsman, R. (1995). Cognitions and stress: Direct and moderating effects on depressive versus externalizing symptoms during the junior high school transition. Journal of Abnormal Psychology, 104, 453-463.

Rueda, B., y Pérez-García, A. (2004). Personalidad y percepción de autoeficacia: Influencia sobre el bienestar y el afrontamiento de los problemas de salud. Revista de Psicopatologia y Psicologia Clínica, 9, 205-219.

Sandín, B. (1999). El estrés psicosocial. Madrid: Klinik.

Sanjuán, P. (1999). Estilo atribucional y depresión: conclusiones y aspectos relevantes. Clínica y Salud, 10, 39-57. 
Sanjuin, P., y Magallares, A. (2005). Spanish adaptation of Attributional Style Questionnaire: Psychometric properties. Trabajo presentado en la VIII European Conference of Psychological Assessment. Budapest (Hungria), 31 de Agosto - 4 de Septiembre. (Acta de Congreso: p. 142).

Sanz, J., y Vázquez, C. (1998). Fiabilidad, validez y datos normativos del Inventario para la depresión de Beck. Psicothema, 10, 303-318.

Southall, D., y Roberts, J. (2002). Attributional style and self-esteem in vulnerability to adolescence depressive symptoms following life stress: A 14-week prospective study. Cognitive Therapy and Research, 26, 563579.

Stiensmeier-Pelster, J. (1989). Attributional style and depressive mood reactions. Journal of Personality, 57, 581-599.
Sweeney, P.D., Anderson, K., y Bailey, S. (1986). Attributional style in depression: A meta-analytic review. Journal of Personality and Social Psychology, 50, 974991.

Vázquez, C., Jiménez, F., Saura, F., y Avia, M.D. (2001). The importance of «importance»: A longitudinal-stress model of depression measuring the importance of the attributions and the impact of stressor. Personality and Individual Differences, 31, 205-214.

Vázquez, C., y Sanz, J. (1997). Fiabilidad y valores normativos de la versión española del Inventario para la depresión de Beck de 1978. Clínica y Salud, 8, 403422.

Wang, C., y Zhang, N. (2005). Personality correlates to attributional style in undergraduates. Chinese Journal of Clinical Psychology, 13, 53-54. 